\title{
Best Practice
}

\section{Robin Fairlie}

is an independent consultant in direct marketing and a Fellow of the IDM

Keywords: NSPCC, segmentation, direct debit, lifetime value

\section{Strategic objectives}

\author{
Need for a step \\ change \\ Robin Fairlie, \\ Co-Editor in Chief, \\ Interactive Marketing, \\ 1 Broadlands Road, \\ London N6 4AE, \\ UK \\ Tel: $44(0) 2083402528$ \\ Fax: 44 (0)20 83408979 \\ E-mail: \\ robinfairlie@highgatelondon. \\ demon.co.uk
}

\section{The NSPCC: The Full Stop Campaign}

\author{
Robin Fairlie
}

Received (in revised form): 12 October 2000

\begin{abstract}
In 2000 the National Society for the Prevention of Cruelty to Children (NSPCC) was the winner of the Gold Award in the IDM/Experian Business Performance awards scheme. This case study derives from the submission of the NSPCC, and their agency WWAV Rapp Collins, to the awards panel.
\end{abstract}

\section{Background}

In 1994 the NSPCC established an independent National Commission of Inquiry to determine whether it was possible to identify all causes of child abuse, and to put a final stop to each one.

The report of the Commission stated that 'Child abuse and neglect can almost always be prevented (in $94 \%$ of cases) provided the will to do so is there.' The NSPCC decided to act on this report, and developed a longterm strategy to end cruelty to children within a generation.

Implementation of this strategy would require both public awareness and support and a greatly increased income.

The NSPCC has been raising money by direct mail since 1980; by 1994 net income from donor direct marketing totalled some $£ 5.2 \mathrm{~m}$. However, there were several problems. First, the growing number of charities using direct marketing media to solicit public donations, coupled with the arrival of the National Lottery, suggested the real possibility of donor exhaustion among the NSPCC's target audience. Secondly, cold mail (prior to 1990 the sole direct medium in use, and still in 1994 a major medium) was becoming increasingly congested, particularly during the NSPCC's favoured pre-Christmas period; this resulted in a decline in the cost-effectiveness of the medium. Thirdly, the emphasis on repeated solicitations to secure repeated donations was not only expensive (between 1994 and 1998 funds raised grew at a much slower rate than fundraising expenditure - see Figure 1 for the charity sector as a whole), but it introduced an undesirable level of uncertainty into budgeting.

The result of this analysis was a realisation that a step change in fundraising was required which would produce a substantial increase in net income, together with a higher level of predictability.

\section{Objectives}

The objectives of the new strategy were: 


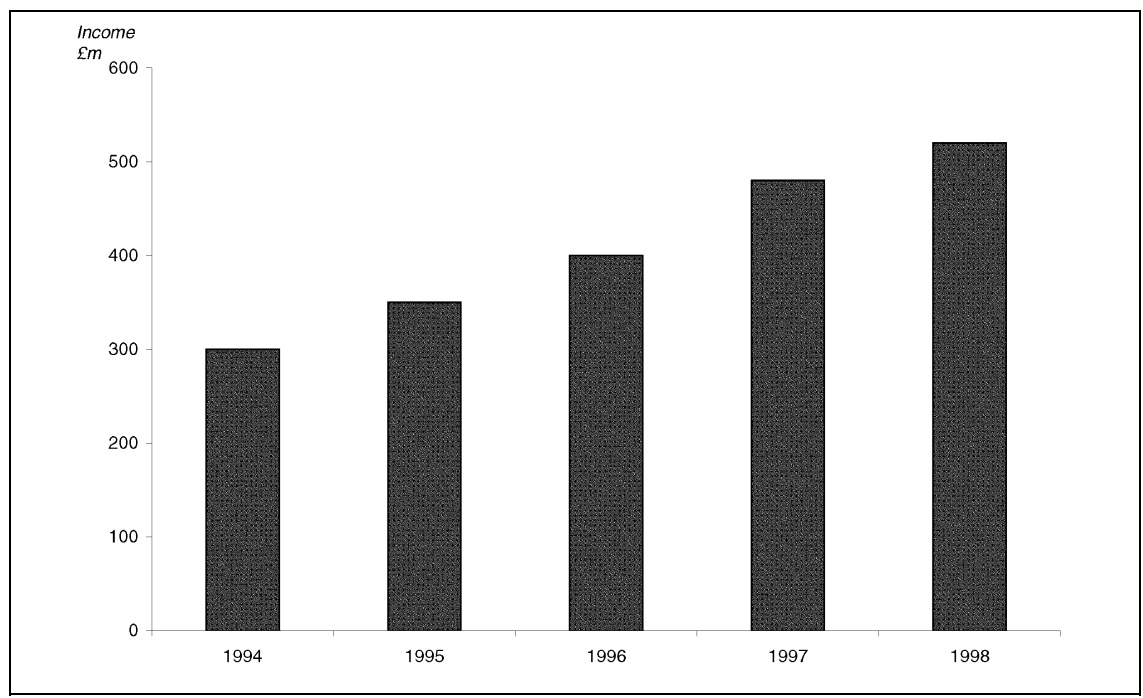

Figure 1: Aggregate charity fundraising expenditure trends 1994-1998

- to maintain cost-effective growth of the existing donor base

- to recruit new donors on the basis of regular, rather than one-off, giving

- to identify the appropriate relationship for each donor in order to maximise lifetime value

- to use the immense exposure generated by the campaign to end cruelty to children in order to maximise donor commitment and longterm net income

- to use direct marketing techniques to enrol support for the NSPCC's campaign to end cruelty to children, and to mobilise action.

\section{Media diversification}

\section{Methodology}

The methods employed to implement each of these objectives were as follows.

\section{Cost-effective growth}

Door drops

In view of the problems discussed above, the NSPCC concluded that reliance upon a single medium (cold direct mail) was no longer an adequate strategy. Accordingly, there has been a continuing effort to diversify into other media. In 1992 an experiment was made in door drops to 500,000 households; by 1998 this had become a regular feature covering up to half the country (13 million households).

\section{DRTV}

Also in 1992 changes in the law enabled charities to fundraise on television for the first time; by 199820 per cent of the NSPCC's recruitment budget was being spent on DRTV. 


\section{The drive for greater predictability of income}

\section{The particular problem of television}

\section{Regular giving}

The importance of all the following schemes in encouraging regular giving lies in the guarantee that they provide of an accrued income, giving much greater security to long-term planning and budgeting.

\section{Banker's order}

The original composition of the direct donor database was built predominantly of donors giving one-off donations, each triggered by a separate appeal. Traditionally, only 40 per cent of these donors could be expected to make a second donation through the donor programme. This meant that a considerable effort was required just to maintain the effective database at a constant level - never mind to increase it. Moreover, direct donor income over any future period was entirely dependent upon response rates to future mailings, which could not be forecast with 100 per cent reliability.

To reduce these problems, the NSPCC sought first to upgrade existing donors by persuading them to give regularly by banker's order. This was followed by rigorous testing to establish the most responsive creative proposition to instigate regular giving at donor recruitment time, via both cold mail and door-drop media.

\section{Direct debit}

As evidence grew in the marketplace of the greater acceptability of direct debits (which had achieved a 42 per cent share of the regular payments market by 1997), the NSPCC changed its approach so as to recruit donors to direct debit rather than banker's order.

\section{Regular giving by DRTV}

The biggest problem area in effecting any change towards greater regular giving was DRTV. It was quickly observable that donors recruited through TV behaved, as a group, in a significantly different way to traditional NSPCC donors; this could be summed up as a lack of attitudinal commitment to the organisation, and consequently an even greater tendency not to respond to further appeals.

To secure regular giving via DRTV was no easy task: it required not merely devising an appropriate creative proposition, but also setting up effective telephone response handling. After considerable testing, success in both these criteria was achieved in 1998, with the use of a new commercial, 'Open Your Eyes', implying the need for long-term help for planning and resourcing, and backed up by 100 per cent live operators; this involved careful media planning to minimise response peaks and troughs. The first full-scale campaign of this type took place in the run-up to Christmas 1999.

\section{Paperless direct debit}

In December 1999 the NSPCC tested the new concept of paperless direct debit as a payment method on DRTV — the first time that a UK charity had done this. 


\section{Prediction of lifetime value}

\section{Lengthy preparation}

Face to face

Finally, the NSPCC has been at the forefront of a new direct response fundraising technique - face-to-face recruitment soliciting a regular giving commitment on the street or the doorstep.

\section{Marketing strategy}

\section{Donor segmentation and lifetime value}

The original donor programme was built on the classic segmentation model using recency, frequency and value to select donors for mailing appeals according to their recent donation history. By 1999, now equipped with a lengthy history for large numbers of donors, it was possible to predict, from quite recent data, the lifetime value of an individual donor. This provided a much more sophisticated segmentation model than the old recency, frequency, value method, and enabled the NSPCC to prioritise their expenditures and maximise individual donor potential. In particular, each donor's lifetime value can be predicted just six months after recruitment; clear segments can be distinguished by propensity to respond to different messages (cash appeals versus regular giving versus upgrades). This in turn enables expenditure to be directed to where it will be most effective.

\section{Donor life cycles}

The NSPCC set out to investigate the hypothesis that donors go through a distinct life cycle, with a different type of communication strategy being appropriate for each different stage in the life cycle. Thus, a year of 'engagement' activity determines whether a donor requires an ongoing relationship (and at what level). Thereafter each donor either enters a maximisation programme, where high-value donors receive premium communications (telephone call, information only etc), while low-value donors receive singleminded anti-attrition messages, or re-enter the recruitment programme.

\section{Donor care}

The emphasis on maximising the return from each donor is mediated, and in the short term constrained, by considerations of donor care - for example the need to respect donors' wishes on how often they should be contacted. Not all communications are appeals: the aim is to give donors a complete picture of the Society's work and achievements.

\section{PR exposure}

The NSPCC campaign to end child abuse and cruelty to children was launched in March 1999 after four years of planning and preparation. In order to take advantage of the publicity caused by this campaign, donor marketing activity was planned in coordination. Specifically:

— huge recruitment in 1997 and 1998 brought the donor file to a peak in 1999 


\section{Taking the pledge}

\section{Shift in patterns of giving}

- this in turn depended on comprehensive testing of recruitment methods in 1996

- maximum attention was given to recruitment of committed givers

- the appeal programme to existing donors was comprehensively tested to allow roll-out in 1999-2000.

\section{Enrolling support}

The key to the Society's 'Full Stop' campaign was to create awareness of the huge problem of child abuse endemic within society, while making the call to action a clear and credible proposition. Thus, while TV and poster advertising raised awareness, direct response activity gave consumers the opportunity to take action.

Traditional direct response media were used to ask people to sign a pledge to end cruelty to children and to become the Society's partners in campaigning, or in fundraising, or in giving. The pledge was distributed by door drop to 23 million households in England, Wales and Northern Ireland. Moreover, all current communications with direct donors reflect a balance between 'Full Stop' campaign messages and the need for longterm income and support for the traditional work of the NSPCC.

\section{Outcomes}

\section{Cost-effective growth}

In 1991 the NSPCC's cold mailings were achieving an acceptable, but unexciting, ROI of 0.9:1. A door-drop test in 1992 achieved an ROI of $1.3: 1$, and the 1998 drop to 13 million households raised over $£ 750,000$. The 1998 DRTV campaign brought returns in excess of 1.1:1.

\section{Regular giving}

By 1994, the NSPCC's drive to obtain banker's orders from donors had brought it an accrued income (that is an annual assured income involving no further marketing cost) of $£ 2 \mathrm{~m}$. Moreover:

- lifetime value of these donors was up to three times that of cash donors $^{1}$

- retention rates of these donors increased from 40 per cent to 90 per cent

- the campaign identified a regular-giving subset among younger, more affluent adults with young families, representing an expansion of the traditional charity market.

Paperless direct debit on DRTV was a great success. Whereas conversion to banker's orders - a two-stage process - had been some 35 per cent, single-stage conversion to direct debit in December 1999 was over 85 per cent.

Overall, committed income has grown sharply in recent years: it now accounts for over 80 per cent of the income from direct donors, and is rising (see Figure 2).

The reduction in recruitment of one-time cash donors has shifted the emphasis of the ongoing donor programme from converting cash donors 


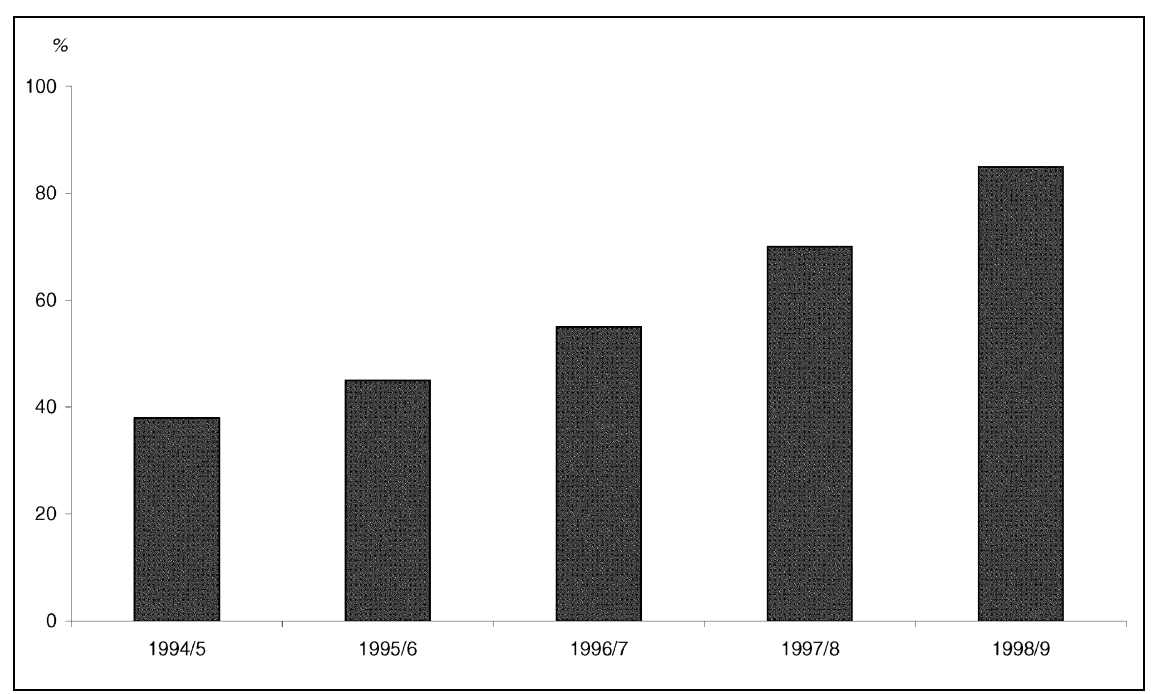

Figure 2: NSPCC committed income trends 1994-1999

Growth of donor numbers and commitment

(to committed giving) to upgrading committed givers to a higher-value donation.

\section{Marketing strategy}

Segmentation by lifetime value has led to positive improvements in response rates of up to 20 per cent. Personalised prompts have resulted in higher average donations, the whole leading to a significant growth in net income from the donor programme, forecast for 2000-2001 at $£ 15 \mathrm{~m}$ (see Figure 3).

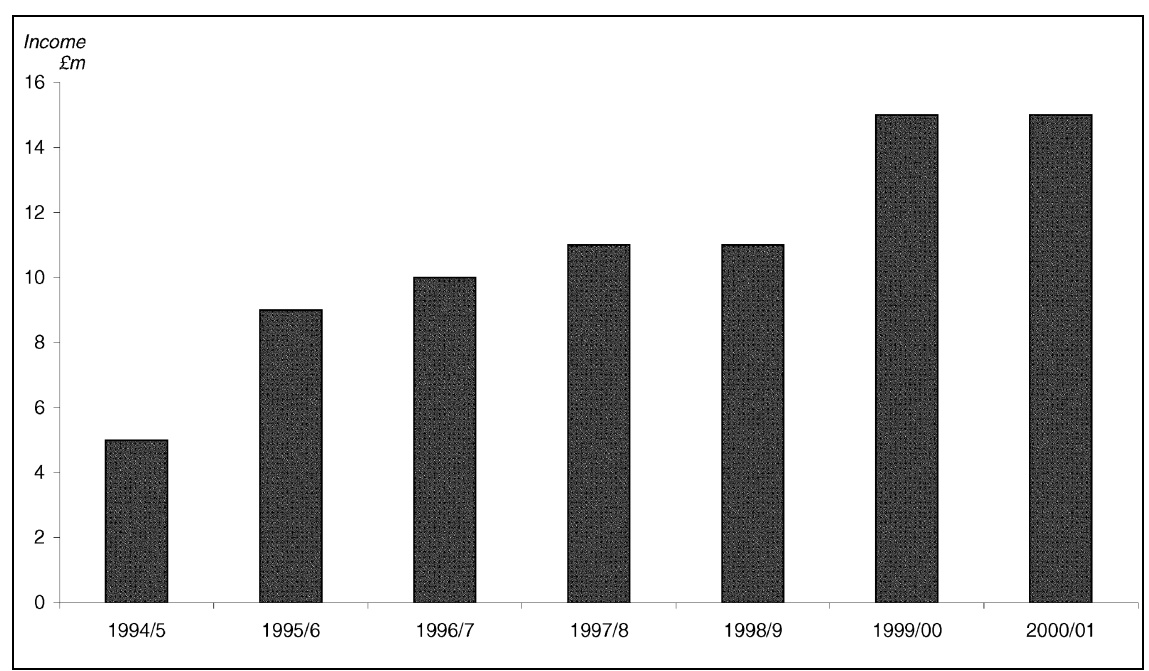

Figure 3: NSPCC net income from donor programme 1994-2001 


\section{PR exposure}

The drive to maximise the donor database in preparation for the launch of the NSPCC 'Full Stop' campaign was extremely successful, with the number of donors rising by 1998-1999 to 330,000, while the number of committed donors reached a quarter of a million (see Figures 4 and 5).

At the height of this campaign 200,000 new donors were being recruited at an acceptable ROI of 0.98:1; with numbers reducing to a more normal level of around 130,000 in 1999-2000, this will improve to 1.42:1.

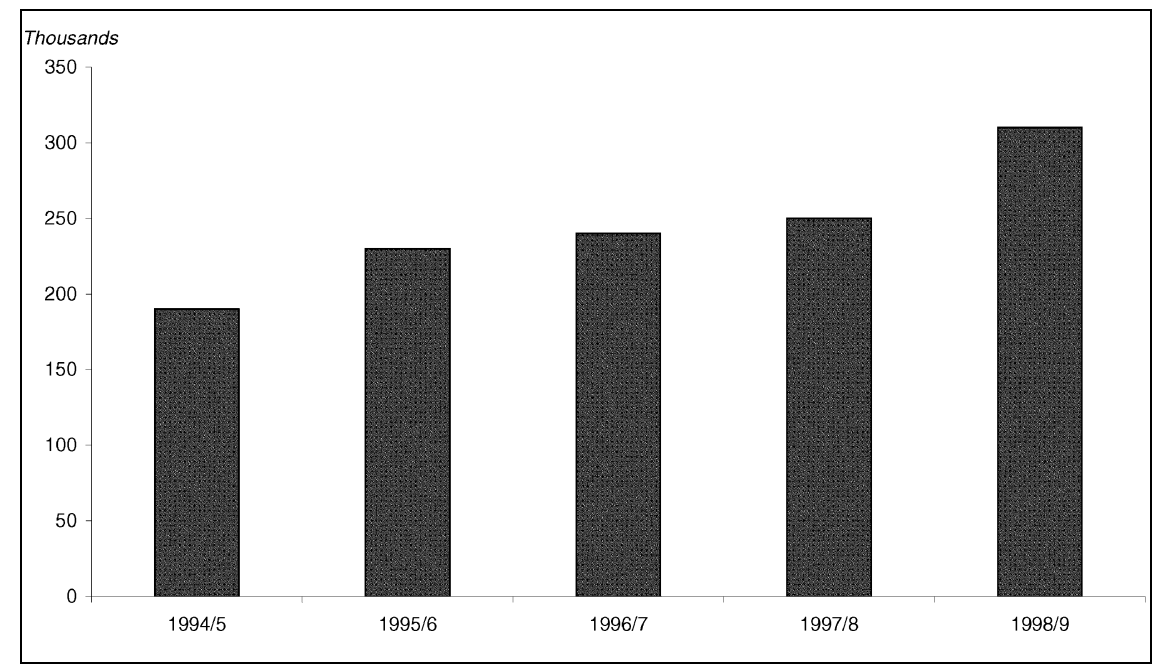

Figure 4: Number of active donors on file

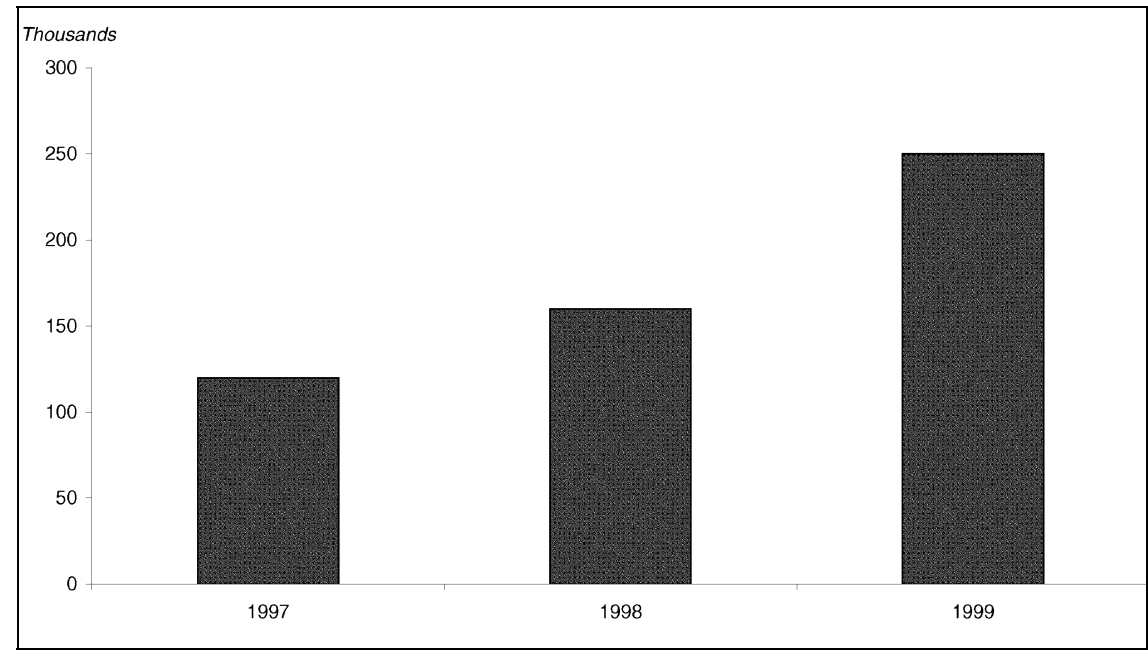

Figure 5: Number of regular-giving donors on file 


\section{Enrolling support}

Over half a million people signed the pledge: 101,584 signed up as partners in fundraising; 126,610 became partners in campaigning; and 90,168 declared themselves partners in giving.

\section{Conclusion}

Getting ahead of the game in charity fundraising is not a once-for-all affair after which one can rest on one's laurels. Having got ahead, one has to stay there. But the NSPCC, in the second half of the 1990s, has put down its marker with an impressive performance - which it will now have to live up to in the century ahead.

\section{Reference}

1. It is, of course, true that these donors are a self-selected group which would certainly have performed better than average in any event — by how much we cannot know. Still, the improvement is impressive. 